\title{
Distribution of facial resemblance in romantic couples suggests both positive and negative assortative processes influence human mate choice
}

\author{
Iris J Holzleitner ${ }^{1}$, Kieran J O’Shea ${ }^{1}$, Vanessa Fasolt ${ }^{1}$, Anthony J Lee ${ }^{2}$, Benedict C Jones ${ }^{1}$ \& \\ Lisa M DeBruine ${ }^{1}$
}

${ }^{1}$ Institute of Neuroscience \& Psychology, University of Glasgow, UK

${ }^{2}$ Division of Psychology, University of Stirling, UK

\begin{abstract}
Author Note
This research was supported by European Research Council grants awarded to LMD (\#647910 KINSHIP), BCJ (\#282655 OCMATE) and AJL (\#705478 MULTIPREF). Dissimilarity data and analysis code are at available at https://osf.io/m9f54/.
\end{abstract}




\begin{abstract}
Previous research suggests that humans show positive assortative mating, i.e. tend to pair up with partners that are similar to themselves in a range of traits, including facial appearance ${ }^{1-12}$. Facial appearance can function as a cue to genetic similarity ${ }^{13}$ and plays a critical role in human mate choice ${ }^{14}$. Evidence for positive assortative mating for facial appearance has largely come from studies showing people can match pictures of couples' faces at levels greater than chance ${ }^{15-}$ ${ }^{19}$ and that facial photographs of couples are rated to look more similar than those of noncouples $^{20,21}$. However, interpreting results from matching studies as evidence of positive assortative mating for facial appearance is problematic, since this measure of perceived compatibility does not necessarily reflect actual physical similarity, and may be orthogonal to, or even negatively correlated with, physical similarity. Even if participants are asked to rate facial similarity directly ${ }^{21}$, it remains unclear which, if any, face shape cues contribute to an increased perception of similarity in romantic couples. Here we use a shape-based assessment of facial similarity to show that the median similarity of long-term couples' face shapes is only slightly greater than that of an age-matched control sample. Moreover, this was driven by the most similar $40 \%$ of couples, while the most dissimilar $20 \%$ of couples actually showed disassortative mating for face shape when compared to the control sample. These data show that a simple measure of central tendency obscures variability in the extent to which couples display assortative or disassortative mating for face shape. By contrast, a more fine-grained analysis that considers the distribution of variation across couples in the extent to which they resemble each other suggests that both positive and negative assortative processes influence human mate choice.
\end{abstract}

Keywords. assortative mating, homogamy, 3D face shape 


\section{Main}

The extent to which romantic couples physically resemble each other is a long-standing question with implications for influential theories of mate choice, such as optimal outbreeding theory $^{22}$. Optimal outbreeding theory acknowledges that mating with closely-related individuals can have a large negative effect on reproductive fitness (i.e., results in less viable offspring), but emphasizes that excessive outbreeding (mating with highly genetically dissimilar individuals), too, can have a negative effect on reproductive fitness ${ }^{23,24}$. Consequently, while folk psychology theories predict that romantic couples will physically resemble each other, optimal outbreeding theory predicts that both assortative and disassortative processes may influence human mate choice.

Several studies have demonstrated that perceptions of facial similarity are very highly correlated with (i.e. nearly indistinguishable from) perceptions of genetic relatedness, demonstrating that facial similarity can function as a cue of genetic relatedness ${ }^{25,26}$. Moreover, facial appearance is known to play a critical role in social interaction, including romantic partner choice ${ }^{14,27,28}$. Consequently, much of the research on the extent to which romantic couples physically resemble each other has investigated facial similarity between romantic partners. While several studies have reported that the faces of romantic partners can be matched at levels greater than chance ${ }^{15-19}$, such results do not necessarily indicate that romantic couples physically resemble each other. For example, matching of romantic couples at levels greater than chance could occur simply because people similar in physical attractiveness are judged more likely to be in a romantic relationship with each other than people who differ in their physical attractiveness ${ }^{29}$ (but $\operatorname{see}^{30}$ ). Moreover, the physical traits associated with attractiveness in men and women are not identical and, in some cases, even opposite. For example, feminine facial features are attractive in women, while masculine facial features are attractive in men (although the extent to which this is the case is disputed ${ }^{31-35}$ ).

This first important limitation of previous work can be avoided entirely by using nonperceptual measures of facial resemblance. One approach for objectively defining and comparing face shape is to assess the position a face occupies in 'face space'. Face space is a multi-dimensional space representing the global face shape dimensions derived from Principal Component Analysis of shape coordinate. Within this multi-dimensional face space, similarity can be quantified as the Euclidean distance between individual faces ( $\mathrm{see}^{36}$ for a recent review).

A second important limitation of previous work on this topic is that it has used measures of 
central tendency to investigate the extent to which couples on average resemble each other. Focusing exclusively on measures of central tendency can, however, obscure important variation in the data ${ }^{37,38}$. This variation is likely to be particularly important in the context of research motivated by optimal outbreeding theory, since optimal outbreeding theory explicitly predicts that both assortative and disassortative processes will influence mate choice. In light of the above, we first used distance in face space to objectively assess the degree of similarity between romantic couples in face shape and compared these scores with controls. We then sought to establish whether there are systematic differences among couples in the extent to which they resembled each other.

First, we calculated shape-dissimilarity scores for 3D scans of 178 couples' faces. Shapedissimilarity scores were the Euclidean distance in a multidimensional face space derived from ten-fold cross-validated PCA of 3D face-shape coordinates. In order to create a control distribution, we identified all possible pairings between each woman and all men in the set who were within five years of her actual partner's age. The median number of control pairings per woman was 71 . We then calculated the dissimilarity score for each control pairing. The median control dissimilarity score for each woman was calculated and is referred to hereon as the control dissimilarity score. Figure 1A shows the distributions of couple and control dissimilarity scores.
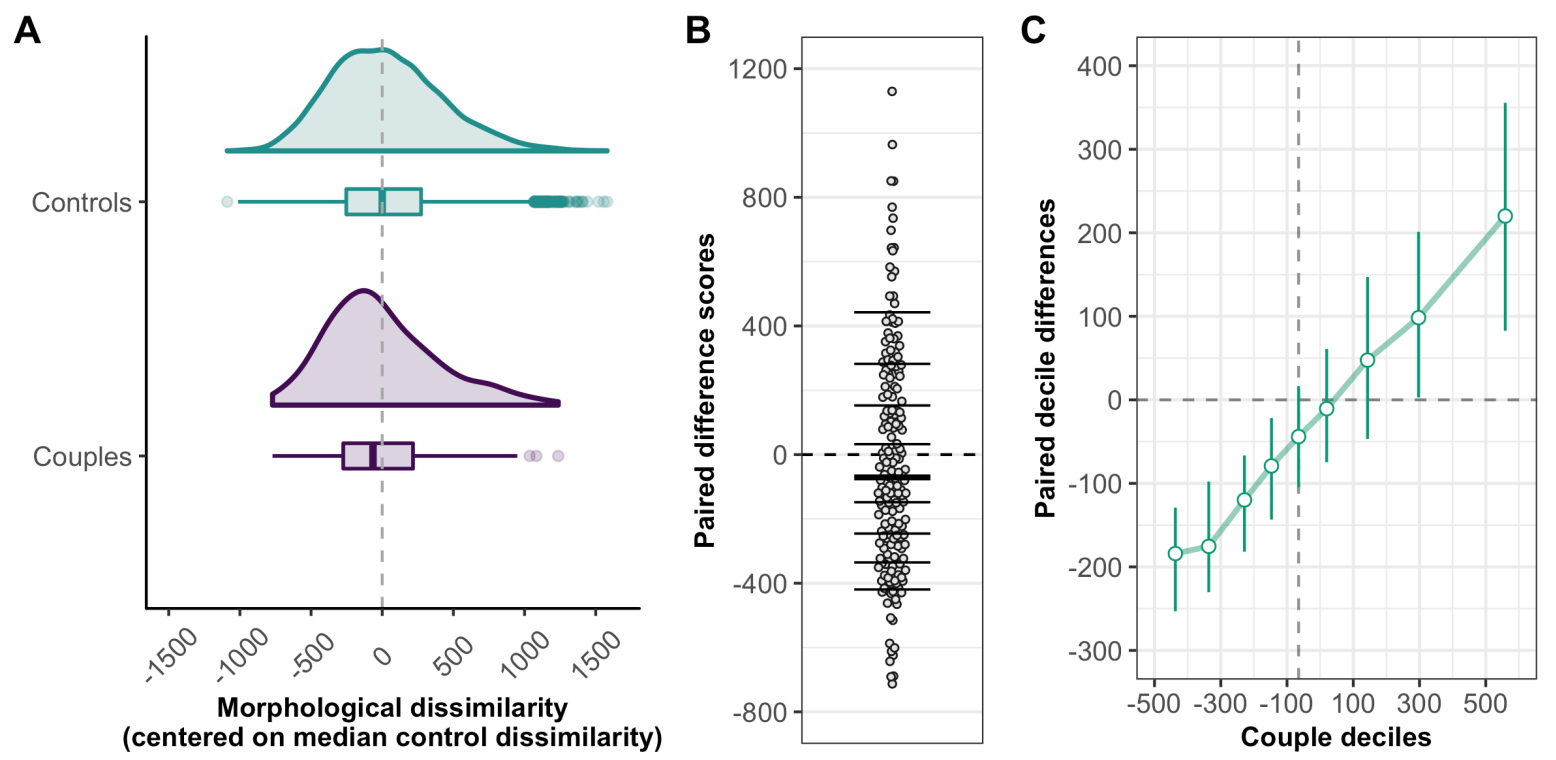

Figure 1. (A) Dissimilarity score distributions of couples (target women + actual partner, $\mathrm{N}=178$ ) and controls (target women + median of age-matched controls, $\mathrm{N}=178$ ). Scores were centered on median control dissimilarity (dashed line). Median couple dissimilarity was marginally smaller than 
median control similarity. (B) Difference strip chart showing the difference scores of similarity between each woman and her actual partner/her median control. The horizontal lines mark the deciles, with the thicker line marking the median. (C) The shift function shows the difference of couples - control for each decile ( $y$-axis) as a function of couple deciles ( $\mathrm{x}$-axis). For each decile difference, the vertical line indicates the $95 \%$ bootstrap confidence interval (1000 samples).

Couple and control dissimilarity scores were initially compared using a paired-samples bootstrapping technique. The median difference score between couple and control dissimilarity was significantly lower than 0 (estimate $=-71, \mathrm{p}=.040$; Figure $1 \mathrm{~B}$ ), suggesting couples are slightly less dissimilar than chance. However, this analysis of central tendency ignores more fine-grained information about the full distribution.

Therefore, we next separated couples into deciles based on their dissimilarity scores. Within each decile, we then compared the couple and control dissimilarity scores and plotted this difference at each decile (Figure 1C). If distributions of couple and control dissimilarity scores were identical, one would expect to see a flat line around 0 for all deciles. If distributions were merely shifted to the left or right, the shift function would show a flat line below or above 0 . Figure $1 \mathrm{C}$ shows that couple dissimilarity was significantly lower than control scores in the first four deciles (i.e., the most similar $40 \%$ of couples) and significantly greater than control scores in the last two deciles (i.e., the most dissimilar $20 \%$ of couples). Thus, while the most similar $40 \%$ of couples show assortative mating for face shape, the most dissimilar $20 \%$ of couples show disassortative mating for face shape. This underlines the limitation of a simple central-tendency comparison of similarity when testing for assortative or disassortative mating.

Analysis of a measure of central tendency showed the type of assortative mating predicted by folk psychology and reported in some previous research. However, the effect was weak. By contrast, analyzing resemblance between couples using deciles, which allows for a far more fine-grained analysis of the distribution of resemblance across couples, showed clear evidence of both assortative and disassortative processes in human mate choice. This finding suggests that individuals may differ in the costs and benefits of assortative vs disassortative mating. Future research could investigate predictors of such individual differences. Not only does the pattern of results found here support an explicit prediction from optimal outbreeding theory (that both assortative and disassortative processes will influence human mate choice), it also highlights the pervasive problem of relying on analyses of measures of central tendency when studying complex behaviors. 


\section{Methods}

Three-dimensional (3D) scans of 178 couples (men: $\mathrm{M} \pm \mathrm{SD}=42.7 \pm 10.3$ years of age; women: $\mathrm{M} \pm \mathrm{SD}=40.3 \pm 10.1$ years of age; relationship length: $\mathrm{M} \pm \mathrm{SD}=16.3 \pm 9.8$ years) were collected using a passive stereo photogrammetry-based 3D camera system (http://di4d.com; see https://osf.io/bvtnj for more information). Participants were scanned individually and seated at a distance of $90 \mathrm{~cm}$ to the camera with a neutral expression. The height of the camera was adjusted to each participant. All participants wore a headband to make sure that face and ears were free from hair and that the hairline was clearly visible. Participants removed glasses, jewelry, and clothing items covering their face or neck.

3D scans were exported as Wavefront OBJ objects. Extraneous parts of face scans (such as captured surface data below the neck) were removed using MeshLab (Visual Computing Lab ISTI-CNR). Each 3D scan was then delineated in MorphAnalyser $2.4^{39}$ using a landmark template of 54 points (see https://osf.io/zsfgr for descriptions of points), aligned to a reference face using Procrustes superimposition, and resampled (i.e., warped) according to this reference face. 3D scans were subsequently converted to PLY format, imported into R v3.5.2 ${ }^{40}$ and subjected to a Generalized Procrustes Analysis using the package Morpho v2.6 ${ }^{41}$. The resulting Procrustes-aligned surface coordinates formed the basis for all subsequent analyses.

Dissimilarity was measured as the Euclidean distance in a multi-dimensional face space derived by Principal Component Analysis (PCA, see supplemental material). To avoid overfitting, we used cross-validation in building the principal component model. The dataset was randomly split into 10 (sex-balanced) folds, one fold excluded, and a principal component analysis conducted on the combined remaining folds. The broken-stick criterion was used to decide how many principal components (PCs) to extract (see Jackson, 1993, for a discussion of the advantages of this criterion). Principal component scores on the selected PCs were then predicted for all 10 folds and saved. This process was repeated for all possible combinations of nine folds, before splitting the data into a new set of 10 folds and repeating all steps again for a total of 10 times, resulting in 100 sets of principal component scores. Next, the Euclidean distance along the multi-dimensional vectors from each PCA model was calculated for both couples and control pairings. Finally, the Euclidean distance for each pairing was averaged across the 100 PCA models, and centered to the median dissimilarity of control pairings. Analysis scripts and dissimilarity data, as well as additional analyses and visualizations, are publicly available on the OSF (https://osf.io/m9f54/). 


\section{References}

1. Agrawal, A., Heath, A. C., Grant, J. D., Pergadia, M. L., Bucholz, K. K., Madden, P. A. F., ... Martin, N. G. (2006). Assortative mating for Cigarette Smoking and for Alcohol Consumption in Female Australian Twins and their Spouses. Behavior Genetics, 36(6), 996-996. https://doi.org/10.1007/s10519-006-9112-5

2. Bovet, J., Barthes, J., Durand, V., Raymond, M., \& Alvergne, A. (2012) Men's Preference for Women's Facial Features: Testing Homogamy and the Paternity Uncertainty Hypothesis. PLoS ONE, 7(11): e49791. https://doi.org/10.1371/journal.pone.0049791

3. Hugh-Jones, D., Verweij, C. J. H., St Pourcain, B., \& Abdellaoui, A. (2016). Assortative mating on educational attainment leads to genetic spousal resemblance for causal alleles. Intelligence, 59, 103-108. https://doi.org/10.1016/j.intell.2016.08.005

4. Keller, M. C., Thiessen, D., \& Young, R. K. (1996). Mate assortment in dating and married couples. Personality and Individual Differences, 21, 217-221. https://doi.org/10.1016/0191-8869(96)00066-9

5. Li, X., Redline, S., Zhang, X., Williams, S., \& Zhu, X. (2017). Height associated variants demonstrate assortative mating in human populations. Scientific Reports, 71), 15689. https://doi.org/10.1038/s41598-017-15864-x

6. Mare, R. D. (1991). Five Decades of Educational Assortative Mating. American Sociological Review, 56(1), 15-32. https://doi.org/10.2307/2095670

7. Pearson, K., \& Lee, A. (1903). On the Laws of Inheritance in Man: I. Inheritance of Physical Characters. Biometrika, 2, 357-462. https://doi.org/10.2307/2331507

8. Silventoinen, K., Kaprio, J., Lahelma, E., Viken, R. J., \& Rose, R. J. (2003). Assortative mating by body height and BMI: Finnish Twins and their spouses. American Journal of Human Biology, 15(5), 620-627. https://doi.org/10.1002/ajhb.10183

9. Stulp, G., Simons, M. J. P., Grasman, S., \& Pollet, T. V. (2017). Assortative mating for human height: A meta-analysis. American Journal of Human Biology, 29(1), e22917. https://doi.org/10.1002/ajhb.22917

10. Yengo, L., Robinson, M. R., Keller, M. C., Kemper, K. E., Yang, Y., Trzaskowski, M., . . . Visscher, P. M. (2018). Imprint of assortative mating on the human genome. Nature Human Behaviour, 2(12), 948-954. https://doi.org/10.1038/s41562-018-0476-3

11. Youyou, W., Stillwell, D., Schwartz, H. A., \& Kosinski, M. (2017). Birds of a Feather Do Flock Together: Behavior-Based Personality-Assessment Method Reveals Personality 
Similarity Among Couples and Friends. Psychological Science, 28(3), 276-284. https://doi.org/10.1177/0956797616678187

12. Zietsch, B. P., Verweij, K. J. H., Heath, A. C., \& Martin, N. G. (2011). Variation in Human Mate Choice: Simultaneously Investigating Heritability, Parental Influence, Sexual Imprinting, and Assortative Mating. The American Naturalist, 177(5), 605-616. https://doi.org/10.1086/659629

13. Crouch, D. J. M., Winney, B., Koppen, W. P., Christmas, W. J., Hutnik, K., Day, T., .. . Bodmer, W. F. (2018). Genetics of the human face: Identification of large-effect single gene variants. Proceedings of the National Academy of Sciences of the United States of America, 115(4), E676-E685. https://doi.org/10.1073/pnas.1708207114

14. Langlois, J. H., Kalakanis, L., Rubenstein, A. J., Larson, A., Hallam, M., \& Smoot, M. (2000). Maxims or myths of beauty? A meta-analytic and theoretical review. Psychological Bulletin, 126, 390-423. https://doi.org/10.1037/0033-2909.126.3.390

15. Bereczkei, T., Gyuris, P., Koves, P., \& Bernath, L. (2002). Homogamy, genetic similarity, and imprinting; parental influence on mate choice preferences. Personality and Individual Differences, 33, 677-690. https://doi.org/10.1016/S0191-8869(01)00182-9

16. Bereczkei, T., Gyuris, P., \& Weisfeld, G. E. (2004). Sexual imprinting in human mate choice. Proceedings of the Royal Society B: Biological Sciences, 271, 1129-1134. https://doi.org/10.1098/rspb.2003.2672

17. Griffiths, R. W., \& Kunz, P. R. (1973). Assortative Mating: A Study of Physiognomic Homogamy. Social Biology, 20, 448-453. https://doi.org/10.1080/19485565.1973.9988075

18. Thiessen, D., Young, R. K., \& Delgado, M. (1997). Social pressures for assortative mating. Personality and Individual Differences, 22(2), 157-164. https://doi.org/10.1016/S0191-8869(96)00181-X

19. Zajonc, R. B., Adelmann, P. K., Murphy, S. T., \& Niedenthal, P. M. (1987). Convergence in the physical appearance of spouses. Motivation and Emotion, 11, 335-346. https://doi.org/10.1007/BF00992848

20. Hinsz, V. B. (1989). Facial resemblance in engaged and married couples. Journal of Social and Personal Relationships, 6(2), 223-229. https://doi.org/10.1177/026540758900600205

21. Wong, Y. K., Wong, W. W., Lui, K. F. H., \& Wong, A. C. N. (2018). Revisiting facial 
resemblance in couples. PLOS ONE, 13(1), e0191456.

https://doi.org/10.1371/journal.pone.0191456

22. Bateson, P. (1978). Early experience and sexual preferences. In J. B. Hutchison (Ed.), Biological determinants of sexual behaviour. London: Wiley.

23. Bateson, P. (1980). Optimal Outbreeding and the Development of Sexual Preferences in Japanese Quail. Zeitschrift fuer Tierpsychologie, 53, 231-244.

https://doi.org/10.1111/j.1439-0310.1980.tb01052.x

24. Partridge, L. (1983). Non-random mating and offspring fitness. In P. Bateson (Ed.), Mate Choice (pp. 227-255). Cambridge, New York, Melbourne: Cambridge University Press.

25. DeBruine, L. M., Smith, F. G., Jones, B. C., Roberts, C. S., Petrie, M., \& Spector, T. D. (2009). Kin recognition signals in adult faces. Vision Research, 49, 38-43. https://doi.org/10.1016/j.visres.2008.09.025

26. Maloney, L. T., \& Dal Martello, M. F. (2006). Kin recognition and the perceived facial similarity of children. Journal of Vision, 6(10), 4-4. https://doi.org/10.1167/6.10.4

27. Little, A. C., Jones, B. C., \& DeBruine, L. M. (2011). Facial attractiveness: evolutionary based research. Philosophical Transactions of the Royal Society B: Biological Sciences, 366, 1638-1659. https://doi.org/10.1098/rstb.2010.0404

28. Rhodes, G. (2006). The evolutionary psychology of facial beauty. Annual Review of Psychology, 57, 199-226. https://doi.org/10.1146/annurev.psych.57.102904.190208

29. Feingold, A. (1988). Matching for Attractiveness in Romantic Partners and Same-Sex Friends: A Meta-Analysis and Theoretical Critique. Psychological Bulletin, 104, 226235. https://doi.org/10.1037/0033-2909.104.2.226

30. Burriss, R. P., Roberts, S. C., Welling, L. M., Puts, D. A., \& Little, A. C. (2011). Heterosexual romantic couples mate assortatively for facial symmetry, but not masculinity. Personality and Social Psychology Bulletin, 37(5), 601-613. https://doi.org/ $10.1177 / 0146167211399584$

31. Said, C. P., \& Todorov, A. (2011). A statistical model of facial attractiveness. Psychological Science, 22, 1183-1190. https://doi.org/10.1177/0956797611419169

32. Scott, I. M. L., Pound, N., Stephen, I. D., Clark, A. P., \& Penton-Voak, I. S. (2010). Does masculinity matter? The contribution of masculine face shape to male attractiveness in humans. PLoS ONE, 5. https://doi.org/10.1371/journal.pone.0013585 
33. Marcinkowska, U. M., Kozlov, M. V., Cai, H., Contreras-Garduno, J., Dixson, B. J., Oana, G. A., ... Rantala, M. J. (2014). Cross-cultural variation in men's preference for sexual dimorphism in women's faces. Biology Letters, 1O(4), 20130850. https://doi.org/10.1098/rsbl.2013.0850

34. Holzleitner, I. J., Lee, A. J., Hahn, A. C., Kandrik, M., Bovet, J., Renoult, J. P., Simmons, D., Garrod, O., DeBruine, L. M., \& Jones, B. C. (2019). Comparing theory-driven and data-driven attractiveness models using images of real women's faces. Journal of Experimental Psychology: Human Perception and Performance. https://doi.org/10.1037/xhp0000685

35. Jones, A. L., \& Jaeger, B. (2019). Biological Bases of Beauty Revisited: The Effect of Symmetry, Averageness, and Sexual Dimorphism on Female Facial Attractiveness. Symmetry, 11(2), 279. https://doi.org/10.3390/sym11020279

36. O'Toole, A. J. (2011). Cognitive and computational approaches to face recognition. In A. J. Calder, G. Rhodes, M. H. Johnson, \& J. V. Haxby (Eds.), The Oxford Handbook of Face Perception (pp. 16-30). Oxford, UK: Oxford University Press.

37. Ritchie, S. J., Cox, S. R., Shen, X., Lombardo, M. V., Reus, L. M., Alloza, C., . . Deary, I. J. (2018). Sex Differences in the Adult Human Brain: Evidence from 5216 UK Biobank Participants. Cerebral Cortex, 28(8), 2959-2975. https://doi.org/10.1093/cercor/bhy109

38. Rousselet, G. A., Pernet, C. R., \& Wilcox, R. R. (2017). Beyond differences in means: robust graphical methods to compare two groups in neuroscience. European Journal of Neuroscience, 46(2), 1738-1748. https://doi.org/10.1111/ejn.13610

39. Tiddeman, B. P., Duffy, N., \& Rabey, G. (2000). Construction and visualisation of threedimensional facial statistics. Computer Methods and Programs in Biomedicine, 63, 920. https://doi.org/10.1016/S0169-2607(00)00072-9

40. R Core Team. (2018). R: A language and environment for statistical computing (Version 3.5.0). Vienna, Austria: R Foundation for Statistical Computing. Retrieved from https://www.R-project.org/

41. Schlager, S. (2017). Morpho and Rvcg - Shape Analysis in R. In Zheng, G., Li, S., \& Szekely, G. (eds.), Statistical Shape and Deformation Analysis (pp. 217-256). Academic Press. https://doi.org/10.1016/B978-0-12-810493-4.00011-0 